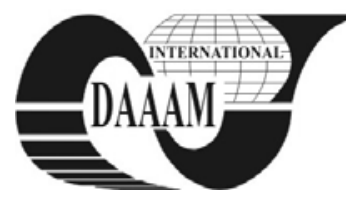

\title{
DESIGNING A GLOBAL FUZZY LOGIC FORECASTER FOR CHAOTIC TIME SERIES
}

\author{
BOUMELLA, N[ora]; IQBAL, S[ohail]; BOULEMDEN, M[ohammed] \& DJOUANI, K[arim]
}

\begin{abstract}
For a robust predictability of chaotic systems, which are very sensitive to initial conditions, we propose in this paper a new approach to design a chaotic system forecaster. Our proposed forecaster is based on a Fuzzy Logic System (FLS). We use the generalized orthogonality principle to tune the forecaster parameters. Substaintially improved performance of the proposed global forecaster is demonstrated on MackeyGlass chaotic time series

Key words: fuzzy logic system, global forecaster, generalized orthogonality principle, caothic systems
\end{abstract}

\section{INTRODUCTION}

Chaotic system trajectories with two nearly initial conditions can differ a lot from each other, which results in long term unpredictability (Kellert, 1993). The inherent property of robustness of a Fuzzy Logic System (FLS) makes it an efficient forecaster for chaotic systems. In this work, we propose a design methodolgy for a fuzzy forecaster for chaotic systems, which essentially deals with the problem of the initial conditions, thus producing a global level of prediction. FLS parameters tuning is essentially equivalent to determining a system that provides an optimal fit to given input-output pairs, with respect to a cost function (Mendel, 2001). A FLS can be expressed as a linear combination of nonlinear Fuzzy Basis Functions (FBF) (Wang \& Mendel, 1992). Our design approach is based on the FBF expansion. We fix the shapes and the parameters of the antecedent Membership Functions (MF) ahead of time. Then we generate the FBFs from training data collected from different initial conditions. This helps the FLS forecaster to be less sensitive to initial conditions. Once the FBFs are computed and collected in different vectors, the aim turn into finding the optimal consequent parameters of the FLS. To do this, we apply a global Generalized Orthogonality Principle (GOP) (Boumella et al., 2009). In order to evaluate the performance of our proposal, we apply it to design a forecaster for Mackey-Glass time-series.

\section{TSK FUZZY LOGIC SYSTEM}

A Takagi-Sugeno Kang (TSK) FLS is represented by fuzzy IFTHEN rules. These rules are the input-output relations of a rule-based fuzzy system. The lth rule of a TSK FLS having $p$ inputs $x_{1} \in \mathbf{X}_{1}, \ldots, x_{p} \in \mathbf{X}_{p}$ and one output $y \in \mathbf{Y}$ is expressed as:

$$
\begin{gathered}
R^{l} \text { : IF } x_{1} \text { is } F_{1}^{l} \text { and } \cdots \text { and } x_{p} \text { is } F_{p}^{l} \\
\text { THEN } y^{l}(x)=c_{0}^{l}+c_{1}^{l} x_{1}+\cdots+c_{p}^{l} x_{p}
\end{gathered}
$$

where $l=1, \cdots, M, c_{0}^{l}(k=0, \cdots, p)$ are the consequent parameters, $y^{l}(\mathbf{x})$ is the output of the $l$ th rule, and $F_{i}^{l}(i=$ $1,2, \ldots, p)$ are antecedent fuzzy sets which are represented by their MFs, $\mu_{F l, i}$. The output of the TSK FLS is obtained by combining the outputs from the $M$ rules in the following form:

$$
y_{T S K}(x)=\frac{\sum_{l=1}^{M} f^{l}(x) y^{l}(x)}{\sum_{l=1}^{M} f^{l}(x)}
$$

where $f^{l}(x)=T_{k=1}^{p} \mu_{F_{k}^{l}}\left(x_{k}\right),(l=1, \ldots, M)$ are the rule firing levels, where $T$ is a $t$-norm operation, i.e., a minimum or product operation, and $\mathbf{x}$ is the input vector (Mendel, 2001). This FLS can be represented as (Boumella et al., 2011):

$$
y_{T S K}(\mathbf{x})=\sum_{l=1}^{M} \sum_{k=0}^{p} c_{k}^{l} \phi_{k}^{l}(\mathbf{x})
$$

where $\phi_{k}^{l}(\mathbf{x})$ is is the $k t h$ Weighted Fuzzy Basis Function (WFBF) of the lth rule that is given as:

$$
\phi_{k}^{l}(\mathbf{x})=x_{k} \phi_{l}(\mathbf{x}),\left\{\begin{array}{l}
l=1, \ldots, M \\
k=0, \ldots, p
\end{array}\right.
$$

with $x_{0} \equiv 1$. and $\phi_{l}=\frac{f^{l}}{\sum_{l=1}^{M} f^{l}}$. This leads to refer to the FLS as a WFBF expansion that provides a natural framework for combining both numerical and linguistic information in a uniform fashion.

\section{CHAOTIC FLS FORECASTER DESIGN BASED ON GLOBAL GOP}

Our design approach is associated with the following problem: Given $M$ linguistic rules of a FLS with $p$ inputs, and $m$ sets of $N_{0}$ input-output numerical data training pairs:

$\left(\mathbf{x}^{(i)}: y^{(i)}\right)^{(k)},\left(i=1, \cdots, N_{0}\right.$ and $\left.k=0, \cdots, m-1\right)$, where $\mathbf{x}$ is the vector input and $y$ is the scalar output of the FLS forecaster for chaotic systems. Each set of data training corresponds to data with different initial conditions. Without the loss of generality, we consider $m=p+1$ different initial conditions, i.e., $(k=0,1, \cdots, p)$. We fix the shapes and the parameters of the antecedent membership functions ahead of time. Then, we use the training data to tune the consequent parameters, i.e., $c_{k}^{l}$.

We rewrite the TSK FLS output (3) using the notation for the $N_{0}$ elements of the $(p+1)$ input-output chaotic training pairs. Then, for each set of the $N_{0}$ values we have:

$$
y_{k}\left(\mathbf{x}^{(i)}\right)=\left\{\begin{array}{ccc}
{\left[\phi_{0}^{1}\left(\mathbf{x}^{(i)}\right)\right.} & \cdots & \left.\phi_{p}^{1}\left(\mathbf{x}^{(i)}\right)\right]\left[\begin{array}{c}
c_{0}^{1} \\
\vdots \\
c_{p}^{1}
\end{array}\right] \\
& +\cdots+ \\
{\left[\phi_{0}^{M}\left(\mathbf{x}^{(i)}\right)\right.} & \cdots & \left.\phi_{p}^{M}\left(\mathbf{x}^{(i)}\right)\right]\left[\begin{array}{c}
c_{0}^{M} \\
\vdots \\
c_{p}^{M}
\end{array}\right]
\end{array}\right.
$$

Where $k=0, \cdots, p, i=k N_{0}+1, \cdots,(k+1) N_{0}$ and $\mathbf{x}^{(i)}=\left[1, x_{1}^{(i)}, \ldots, x_{p}^{(i)}\right]$. We take $\vec{C}=\left(\begin{array}{ccc}c_{0}^{1} & \cdots & c_{p}^{M}\end{array}\right)^{T}$ Then, in matricial form, (5) becomes: 


$$
\overrightarrow{y_{k}}=\boldsymbol{\Phi}^{(\boldsymbol{k})} \overrightarrow{\boldsymbol{C}}
$$

Where $\boldsymbol{\Phi}^{(k)}$ is the kth Weighted Basis Function Matrix (WBFM). Now, our aim is to find the elements of the vector $\overrightarrow{\boldsymbol{C}}$, i.e., the consequent parameters which minimize the norm errors between the vectors $\overrightarrow{y_{k}}$ and the corresponding columns of the WBFM, $\boldsymbol{\Phi}^{(k)}$. We apply the GOP design (Boumella et al., 2009). In this case, the $(p+1)$ error vectors $\overrightarrow{y_{k}}-\boldsymbol{\Phi}^{(k)} \overrightarrow{\boldsymbol{C}}$ must be perpendicular to all of the WFBM columns. Solving for $\overrightarrow{\boldsymbol{C}}$ gives:

$$
\overrightarrow{\boldsymbol{C}}_{\text {opt }}=\left\{\begin{array}{c}
{\left[\begin{array}{ccc}
{\left[\boldsymbol{\Phi}^{(0)}\right.} & \cdots & \boldsymbol{\Phi}^{(p)}
\end{array}\right]\left[\begin{array}{c}
\boldsymbol{\Phi}^{(0)} \\
\vdots \\
\boldsymbol{\Phi}^{(p)}
\end{array}\right]} \\
{ }^{(0)} \\
{\left[\begin{array}{lll}
\boldsymbol{\Phi}^{(0)} & \cdots & \boldsymbol{\Phi}^{(p)}
\end{array}\right]\left[\begin{array}{c}
\overrightarrow{y_{0}} \\
\vdots \\
\overrightarrow{y_{p}}
\end{array}\right]}
\end{array}\right.
$$

Where $\overrightarrow{\boldsymbol{C}}_{\text {opt }}$ contains the $(p+1)$-dimensional vectors, containing the optimal consequent parameters of the chaotic TSK FLS forecaster for each rule.

\section{APPLICATION TO A CHAOTIC TIME-SERIES GLOBAL FORECASTING}

In this section, we design a global fuzzy forecaster for a Mackey-Glass time-series:

$$
\frac{d s(t)}{d t}=\frac{0.2 s(t-\tau)}{1+s^{10}(t-\tau)}-0.1 s(t)
$$

We use four antecedents ( $p=4)$ for forecasting, $s(k-18)$, $s(k-12), s(k-6)$ and $s(k)$, to predict $s(k+6)$.

The training data are obtained by simulating (8) for $\tau=20$. The resulting equation requires 20 initial values because of the timeunit delay. We choose the initial values randomly $(p+1)$ times. This results in five very different trajectories for the same timeseries, $s_{0}, \cdots, s_{4}$. Then, we use the samples: $s_{0}(1001) \sim s_{0}(1124), s_{1}(1101) \sim s_{1}(1224), s_{2}(1201) \sim$ $s_{2}(1324) s_{3}(1301) \sim s_{3}(1424)$ and $s_{4}(1401) \sim s_{4}(1524)$ to design the global forecaster. To demonstrate the effectiveness of the approach, we design a local forecaster, i.e., with only one set of initial conditions and then we compare the obtained results with those obtained with the global forecaster. The local forecaster is designed by using the samples $s_{0}(1001) \sim s_{0}(1124)$. For both forecasters the antecedent parameters (the gaussian means and standard deviations) are fixed according to the mean and the standard deviation of the corresponding training samples. To test the designed forecasters we generate again different initial conditions randomly, we will refer to the corresponding Mackey-Glass time-series as $s_{5}(k)$.

Then we use the samples $s_{5}(1501) \sim S_{5}(1524)$ for the test. Figure 1 shows the outputs of both forecasters to the test samples generated with another different initial conditions, $s_{5}$. To evaluate and compare the performance of both designs we use the following Root Mean Square Error (RMSE):

$$
R M S E=\sqrt{\frac{1}{500} \sum_{k=1519}^{2018}\left(s_{5}(k+6)-y\left(\mathbf{x}^{(k)}\right)\right)^{2}}
$$

where $\mathbf{x}^{(k)}=\left[s_{5}(k-18), s_{5}(k-12), s_{5}(k-6), s_{5}(k)\right]$ and $y\left(\mathbf{x}^{(k)}\right)$ is the global or local

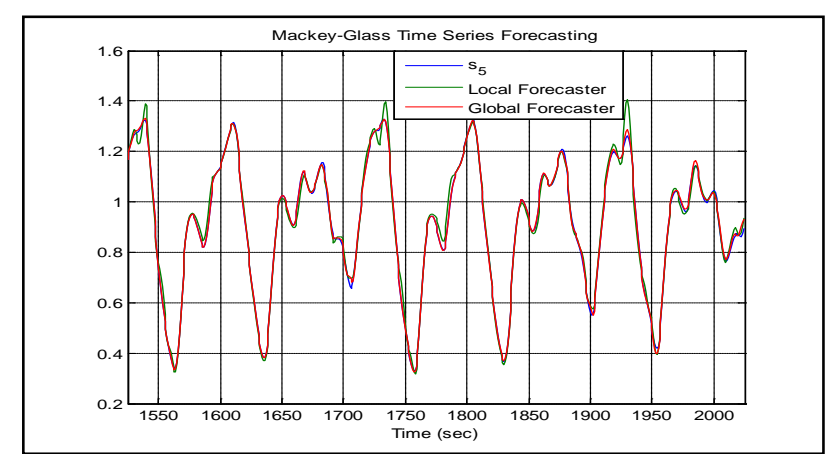

Fig. 1. Outputs of global and local time-series Forecasters.

forecaster output. The RMSE values of both designs are $R M S E_{\text {glbl }}=0.0077$ and $R M S E_{l c l}=0.0252$. Note that the the RMSE obtained by the global-designed forecaster is $31 \%$ of the RMSE obtained by the local-designed-one. There is a substantial improvement in performance for the globaldesigned forecaster over the local-designed one. This improvement is due to the fact that for the global-designed forecaster we used different initial conditions which led the forecaster to be less sensitive to the variation in the initial conditions.

\section{CONCLUSION}

As part of this paper we have presented a forecaster based on fuzzy logic system to globally forecast chaotic time-series. To force the forecaster to be less sensitive to initial conditions, we used training data obtained from a chaotic time-series simulated with different initial conditions, thus producing a more global level of prediction. We applied our proposal to forecast a Mackey-Glass time-series with a delay exhibiting chaos. The obtained results have shown that the fact to take into account the variation in the initial conditions in the design stage makes the forecaster more robust and less sensitive to initial conditions.

\section{REFERENCES}

Boumella, N.; Djouani, K. \& Iqbal, S. (2009). A New Design of Fuzzy Logic Controller Based on Generalized Orthogonality Principle, International Symposium on Computational Intelligence in Robotics and Automation, Daejon, ISBN 978-1-4244-4808-1, pp. 497-502, IEEE, Korea

Boumella, N.; Djouani, K. \& Boulemden, M. (2011). On an Interval Type-2 TSK FLS A1-C1 Consequent Parameters Tuning, IEEE T2FUZZ Symposium on Advances in Type-2 Fuzzy Logic Systems, ISBN: 978-1-61284-077-2 , pp 150156, IEEE, France

Kellert, S. H. (1993). In the Wake of Chaos: Unpredictable Order in Dynamical Systems, University of Chicago Press, ISBN 0-226-42976-8, Chicago

Mendel, J. M. (2001). Uncertain Rule-Based Fuzzy Logic Systems: Introduction and New Directions, Prentice-Hall PTR, ISBN 0-13-040969-3, Upper-Saddle River, NJ

Wang, L. \& Mendel, J. M. (1992). Fuzzy Basis Functions, Universal Approximation, and Orthogonal Least Squares Learning, IEEE Trans. Neural Networks Vol. 3, No. 5, pp. 807-814 